\title{
Atherosclerotic Vascular Events in a Multinational Inception Cohort of Systemic Lupus Erythematosus (SLE)
}

\author{
M. B. UROWITZ, D. GLADMAN, D. IBAÑEZ 1 , S.C. BAE ${ }^{2}$, J. SANCHEZ-GUERRERO ${ }^{3}$, C. \\ GORDON $^{4}$, A. CLARKE, S. BERNATSKY ${ }^{5}$, P.R. FORTIN ${ }^{6}$, J. G. HANLY ${ }^{7}$, D. WALLACE ${ }^{8}$, D. \\ ISENBERG, A. RAHMAN ${ }^{9}$, G.S. ALARCÓN ${ }^{10}$, J. MERRILL ${ }^{11}$, E. GINZLER ${ }^{12}$, M. $^{2}$ \\ KHAMASHTA $^{13}$, O. NIVED, G. STURFELT ${ }^{14}$, I. N. BRUCE ${ }^{15}$, K. STEINSSON $^{16}$, S. MANZI ${ }^{17}$, \\ R.. RAMSEY-GOLDMAN ${ }^{18}$, M A. DOOLEY ${ }^{19}$, A. ZOMA ${ }^{20}$, K. KALUNIAN ${ }^{21}$, M. RAMOS ${ }^{22}$, R. F. \\ VAN VOLLENHOVEN ${ }^{23}$, C. ARANOW ${ }^{24}$, T. STOLL ${ }^{25}$, M. PETRI ${ }^{26}$, and P. MADDISON ${ }^{27}$ FOR \\ THE SYSTEMIC LUPUS INTERNATIONAL COLLABORATING CLINICS
}

\begin{abstract}
${ }^{1}$ M. B. Urowitz, MD, D. Gladman, MD, D. Ibañez, MSc: Toronto Western Hospital, Toronto, ON, Canada ${ }^{2}$ S. C. Bae, MD: Hospital for Rheumatic Diseases and Hanyang University Medical Center, Seoul, Korea ${ }^{3}$ J. Sanchez-Guerrero, MD: Instituto Nacional de Ciencias Medicas y Nutricion, Mexico City, Mexico ${ }^{4}$ C. Gordon, MD: Medical School, University of Birmingham, Birmingham, UK ${ }^{5}$ A. Clarke, MD, S. Bernatsky, MD: Montreal General Hospital and McGill University Health Centre, Montreal, Quebec, Canada ${ }^{6}$ P. R. Fortin, MD: Toronto Western Hospital and University of Toronto, Toronto, Ontario, Canada ${ }^{7}$ J. G. Hanly, MD: Queen Elizabeth II Health Sciences Centre and Dalhousie University, Halifax, Nova Scotia, Canada ${ }^{8}$ D. J. Wallace, MD: Cedars-Sinai/David Geffen School of Medicine at University of California, Los Angeles, USA ${ }^{9}$ D. Isenberg, MD, A. Rahman, MD: University College, London, UK ${ }^{10} \mathrm{G}$. S. Alarcón, MD, MPH: University of Alabama at Birmingham, USA ${ }^{11} \mathrm{~J}$. T. Merrill, MD: Oklahoma Medical Research Foundation, Oklahoma City, USA ${ }^{12}$ E. Ginzler, MD: State University of New York Downstate Medical Center, Brooklyn, USA ${ }^{13}$ M. Khamashta, MD: St. Thomas' Hospital and King's College London School of Medicine, London, UK ${ }^{14}$ O. Nived, MD, G. Sturfelt, MD: University Hospital Lund, Lund, Sweden ${ }^{15} \mathrm{I}$. N. Bruce, MD: University of Manchester, Manchester, UK ${ }^{16} \mathrm{~K}$. Steinson, MD: Landspitali University Hospital, Reyjkavik, Iceland ${ }^{17} \mathrm{~S}$. Manzi, MD: University of Pittsburgh School of Medicine, Pittsburgh, Pennsylvania, USA ${ }^{18}$ R. Ramsey-Goldman, MD: Northwestern University and Feinberg School of Medicine, Chicago, Illinois, USA ${ }^{19}$ M. A. Dooley, MD: University of North Carolina, Chapel Hill, USA ${ }^{20}$ A. Zoma, MD: Hairmyres Hospital, East Kilbride, UK ${ }^{21}$ K. Kalunian, MD: University of California, San Diego School of Medicine, San Diego, USA ${ }^{22}$ M. Ramos, MD: Unidad de Enfermedades, Barcelona, Spain ${ }^{23}$ R.F. Van Vollenhoven, MD: The Karolinska Institute, Stockholm, Sweden ${ }^{24}$ C. Aranow, MD: Columbia University Medical Center, New York, USA ${ }^{25}$ T. Stoll, MD:Kantonsspital, Schaffhausen, Switzerland ${ }^{26}$ M. Petri, MD: Johns Hopkins University School of Medicine, Baltimore, Maryland, USA ${ }^{27}$ P. Maddison, MD: North West Wales NHS Trust, Bangor, Wales
\end{abstract}

\footnotetext{
AUTHOR CONTRIBUTIONS

Dr. Urowitz had full access to all of the data in the study and takes responsibility for the integrity of the data and the accuracy of the data analysis.

Study design: Urowitz, Gladman.

Acquisition of data. Urowitz, Gladman, Ibanez, Bae, Sanchez-Guerron, Gordon, Clarke, Bernatsky, Fortin, Hanly, Wallace, Isenberg, Rahman, Alarcon, Merrill, Ginzler, Khamashta, Nived, Sturfelt, Bruce, Stiensson, Manzi, Ramsey-Goldman, Dooley, Zoma,

Kalunian, Font, Ramos, Van Vollenhoven, Aranow, Stoll, Pietri, Maddison.

Analysis and Interpretation of Data. Urowitz, Gladman, Ibanez.

Manuscript Preparation. Urowitz, Gladman, Ibanez.

Statistical Analysis. Ibanez.
} 


\section{Abstract}

Objective-To describe vascular events during an 8 year follow-up in a multicentre SLE inception cohort and their attribution to atherosclerosis.

Methods-Clinical data including co-morbidities are recorded yearly. Vascular events are recorded and attributed to atherosclerosis or not. All events met standard clinical criteria. Factors associated with atherosclerotic vascular events were analysed using descriptive statistics, t-tests and $\chi^{2}$. Stepwise multivariate logistic regression was used to assess the association of factors with vascular events attributed to atherosclerosis.

Results-Since 2000, 1249 patients have been entered into the cohort. There have been 97 vascular events in 72 patients. These include: myocardial infarction (13), angina (15), congestive heart failure (24), peripheral vascular disease (8), transient ischemic attack (13), stroke (23), pacemaker insertion (1). Fifty of the events were attributed to active lupus, 31events in 22 patients were attributed to atherosclerosis, and 16 to other causes. Time from diagnosis to first atherosclerotic event was $2.0 \pm 1.5$ years. Compared to patients followed for 2 years without atherosclerosis events (615), at enrolment patients with AVE were more frequently Caucasian, male, older at diagnosis of SLE, obese, smokers, hypertensive and had a family history of coronary artery disease. On multivariate analysis only male gender and older age at diagnosis were associated factors.

Conclusion-In an inception cohort with SLE followed for up to 8 years there were 97 vascular events but only 31 were attributable to atherosclerosis. Patients with atherosclerotic events were more likely to be male and to be older at diagnosis of SLE.

\section{Background}

Since the first description of the bimodal mortality pattern of systemic lupus erythematosus (SLE) showing that coronary artery disease (CAD) is a significant cause of morbidity and mortality in this condition, multiple studies have addressed the nature of these clinical outcomes and the associated risk factors ${ }^{1}$. However, the exact incidence and prevalence of atherosclerotic events is unknown. A few studies have attempted to identify risk factors for CAD in lupus $2,3,4,5$. To date these studies have found elevated total cholesterol and older age at diagnosis of SLE to be significantly associated with CAD. Other factors implicated in one or two studies have been hypertension ${ }^{2,3}$, previous cardiac involvement with SLE, obesity $^{2}$, and longer duration of corticosteroid use ${ }^{3}$. However, lupus populations vary with regard to organ involvement and it has not been possible to adequately address these variables in the past, given the limited power of previous studies. Furthermore, incident SLE subjects were rarely studied.

To address this complex clinical problem with appropriate epidemiologic tools in a relatively uncommon disease such as SLE would be impossible without a concerted international effort. The Systemic Lupus Erythematosus International Collaborating Clinics (SLICC) is a group of rheumatologists and methodologists from 27 international centres who have a particular interest and expertise in SLE. The co-operating centres all follow large cohorts of SLE patients in standardized databases. The group has been working together since $1987^{6}$. SLICC has developed an international registry of newly diagnosed SLE patients in order to provide a large diverse population to carry out a prospective longitudinal study. One of the major objectives of the study is to determine the prevalence and type of atherosclerotic vascular disease in SLE and to identify associated risk factors. The SLICC group has shown that a significant number of coronary artery disease risk factors are present within the first year of disease ${ }^{7}$. 
The aims of this SLICC investigation were to describe the vascular events occurring during the first 8 years of follow-up and to attribute the vascular events to atherosclerosis.

\section{Methods}

\section{The SLICC registry for atherosclerosis (SLICC-RAS)}

Twenty-six SLICC centres from 11 countries, in North America, Europe and Asia participated in this study. An inception cohort of 1249 SLE patients has been assembled according to a standardized protocol between 2000 and 2008 to study risk factors for atherosclerosis. Data collected includes clinical and laboratory features of lupus, CAD risk factors and atherosclerotic outcomes based on a glossary of established definitions.

\section{Patients}

Patients are enrolled in the Registry within 15 months of their date of diagnosis, based on $\geq 4$ American College of Rheumatology (ACR) classification criteria ${ }^{8}$. Demographic, clinical and laboratory features of lupus, CAD risk factors and atherosclerotic outcome and attribution data collected are submitted to the coordinating centre at the University of Toronto at enrolment and once annually. Disease activity is measured by SLEDAI-2 $\mathrm{K}^{9}$ and adjusted mean SLEDAI-2K (AMS) ${ }^{10}$. Damage is measured by the SLICC/ACR damage index ${ }^{11,12}$.

Definitions of atherosclerotic outcomes and attribution-Myocardial infarction defined on the basis of definite electrocardiographic (ECG) abnormalities or symptoms of chest pain with probable ECG abnormalities and abnormal cardiac enzymes, or typical symptoms and abnormal cardiac enzymes, or fresh myocardial infarction or coronary occlusion at post mortem ${ }^{13}$.

Attribution to atherosclerosis: SLE inactive at the time of the event (based on the treating physician opinion and not associated with increase or new therapy); and any of typical atherosclerotic changes on angiogram; evidence of atherosclerosis elsewhere (cerebrovascular, peripheral vascular). Examinations to confirm these criteria were at the discretion of the treating physician.

Angina: severe pain or discomfort over the upper or lower sternum or anterior left chest and left arm on exertion, relieved at rest. Attribution to atherosclerosis: As above.

Congestive heart failure: Diagnosed clinically or on chest x-ray.

Attribution to atherosclerosis: As above

Insertion of pace maker attributed to atherosclerosis as above.

Intermittent claudication: classic symptoms including cramping pain and weakness in the legs especially the calves on walking that disappears after rest.

Attribution to atherosclerosis: SLE inactive at the time of the event (based on the treating physician opinion and not associated with increase or new therapy); typical atherosclerotic changes on angiogram; evidence of atherosclerosis elsewhere (cardiac, cerebral vascular)

Transient ischemic attack (TIA): transient and completely reversible neurological deficit with complete recovery within 24 hours. 
Attribution to atherosclerosis: SLE inactive at the time of the event (based on the treating physician opinion and not associated with increase or new therapy); typical atherosclerotic changes on angiogram; evidence of atherosclerosis elsewhere (cardiovascular, peripheral vascular)

Stroke: irreversible or partially reversible motor and/or sensory deficits of sudden or recent onset on the basis of vascular occlusion or insufficiency including complete or incomplete stroke or stroke in evolution.

Attribution to atherosclerosis: as above.

\section{Statistical analysis}

Data presented for descriptive purposes are mean \pm standard deviation (sd) for continuous variables as well as percentages for categorical variables. Patients with atherosclerotic vascular events were compared to patients with no atherosclerotic vascular events using ttests and chi-squared tests. Stepwise logistic regression analysis was used to determine the contribution of multiple putative risk factors and their association to the presence of vascular events attributed to atherosclerosis. Adequacy of model fit was established with Hosmer and Lemeshow goodness-of-fit test. Factors included in the model were those which were significantly different in the univariate analysis. Finally, comparisons between patients with vascular events attributed to atherosclerosis to patients with events attributed to SLE were made using non-parametric tests due to the small sample size, including Fisher's exact test and Wilcoxon rank sum test. A multivariate analysis was not conducted with the group of patients because of sample size limitations.

\section{Results}

\section{Characteristics of the SLICC-RAS cohort at enrolment}

Of the 1249 patients enrolled, 1117 (89\%) were female, 49\% were Caucasian, 15\% Black, $16 \%$ Hispanic, $16 \%$ Asian and $4 \%$ other. The mean age at presentation was 34 years, and mean disease duration at entry to the cohort was 5.5 months. The average SLEDAI-2K was 5.5 and the majority were already on corticosteroid and antimalarial therapy (Table 1).

\section{Vascular events}

Ninety-seven vascular events occurred in 72 patients. The majority of the events (61) were cardiovascular in nature including myocardial infarction -13 ; angina -15 ; congestive heart failure -24; pacemaker insertion -1 ; peripheral vascular disease -8 . Thirty six events were cerebrovascular, including transient ischemic attacks -13 and stroke -23 .

\section{Attribution of vascular events}

Of the 97 vascular events only 31 (in 22 patients) were attributed to atherosclerosis and occurred at an average ( \pm sd) of $2.0( \pm 1.5)$ years after entry into the cohort. Of the fourteen events due to myocardial infarction, intermittent claudication or transient ischemic attack 10 were confirmed by imaging studies. Fifty of the 97 vascular events were attributed to active lupus, and 16 to other causes (Table 2).

\section{Baseline comparison of patients with and without atherosclerotic vascular events followed for at least 2 years}

Baseline variables for the 22 patients with atherosclerotic vascular events were compared to 615 SLE patients who did not have atherosclerotic vascular events and were followed for at least 2 years. A two year follow up for the controls was chosen since the atherosclerotic 
vascular events occurred at an average of 2 years after entry into the cohort. Males make up $11 \%$ of the inception cohort but make up $41 \%$ of the patients with atherosclerotic events. Other features of patients with atherosclerotic events are Caucasian ethnicity, older age at diagnosis of SLE, hypertensive, obese, smokers, and with a positive family history for CAD (Table 3). There was no difference at baseline in the frequency of hypercholesterolemia, diabetes, use of corticosteroids, antimalarials or immunosuppressive drugs between patients with and without atherosclerotic vascular events. SLEDAI-2K at presentation as well as AMS at year 2 were similar in both groups. A stepwise multivariate analysis including the items which were significant on the univariate analyses revealed only older age at diagnosis (OR 1.08 95\% CI $(1.05,1.11)$ ) and male gender (OR 3.67, 95\% CI $(1.41,9.52)$ ) as independently associated with atherosclerotic vascular events. Hosmer and Lemeshow goodness-of-fit test $\mathrm{p}$ value $=0.59$, indicating that the model fit is adequate .

\section{Patients with vascular events due to lupus compared to those with atherosclerotic vascular events}

We then compared patients with atherosclerotic events to those with vascular events due to lupus (Table 4). Male gender, older age at diagnosis, Caucasian race and use of antimalarials at baseline were associated with atherosclerotic vascular events.

\section{Discussion}

This study reports on atherosclerotic events in an international inception cohort of patients with SLE. Of the 97 vascular events documented among the 1249 patients, only 31 events were attributable to atherosclerosis. These events occurred within 2 years of entry into the cohort, or approximately 2.5 years from diagnosis. In long-term follow up studies the prevalence of atherosclerotic vascular events varies from $8-13 \% 14,15$ and usually occurs at a mean of $8-9$ years from diagnosis ${ }^{1,15}$.

This study describes atherosclerotic vascular events in 22 of 1249 (1.8\%) patients. As previously noted, SLE patients with atherosclerotic vascular events tend to be older at SLE diagnosis than those without these events ${ }^{3,2}$. In this study patients with atherosclerotic vascular events were twenty years older than those without events, accounting in part for the earlier onset of atherosclerotic vascular events (within 2.5 years of SLE diagnosis) in this inception cohort. In univariate analyses patients with vascular events due to atherosclerosis were more likely to have the classic risk factors for atherosclerotic disease than patients without atherosclerotic vascular events who had been followed for at least 2 years. They were more likely to be male, older, hypertensive, obese, smoker and have a family history of CAD. Disease activity at presentation and over the first two years was similar in patients who had atherosclerotic vascular events and patients who did not. There was also no difference in the baseline use of medications between the two groups. However, in multivariate analysis only male gender and older age remained independently associated with atherosclerotic vascular events.

In the Baltimore study of 229 patients, 29 events were reported. In a multivariate analysis, patients with events had higher cholesterol, history of hypertension, a longer mean SLE duration and greater duration and use of corticosteroids (Table 5). However, although the cohort was followed for 4 years, it was a prevalent cohort with mean SLE disease duration in those with atherosclerotic vascular events of 12 years compared to 8 years in those without $^{2}$. In the Pittsburgh cohort, also a prevalent cohort of 498 women, there were 33 events. The risk factors identified in a multivariate analysis included older age at diagnosis, longer SLE duration, longer duration of corticosteroid use, hypercholesterolemia and postmenopausal status. In this cohort, the SLE disease duration in those with atherosclerotic vascular events was 13.0 years compared to 10.0 in those without ${ }^{3}$. In the LUMINA cohort, 
which includes patients within 5 years of diagnosis, of 546 patients there were 34 vascular events including angina, myocardial infarction, vascular interventions, strokes and peripheral vascular events. Risk factors identified in multivariate analysis included older age, current smoking status, longer follow-up time, elevated C-reactive protein and the presence of antiphospholipid antibody ${ }^{16}$. In the Toronto cohort, of 1087 patients who did not have atherosclerotic vascular events prior to entry to the cohort, 144 atherosclerotic vascular events were documented. In a multivariate analysis of risk factors for atherosclerotic vascular events, neuropsychiatric disease, vasculitis and number of Framingham CAD risk factors were associated with coronary artery events ${ }^{15}$. In the inception patients in the Toronto Cohort which included 561 patients seen within 1 year of diagnosis and followed through 9 years of follow-up $9.6 \%$ had atherosclerotic vascular events. A multivariate analysis of risk factors for atherosclerotic vascular events revealed neuropsychiatric disease and smoking to be associated with atherosclerotic vascular events ${ }^{15}$.

In the two inception cohorts with disease duration at entry of less than 1 year (the Toronto inception cohort and the current SLICC/RAS cohort study), the classic Framingham risk factors were not clearly independently associated with atherosclerotic vascular events, as there has been a shorter exposure to these risk factors perhaps mitigating their impact. It is possible that over time these risk factors will exert their influence. Furthermore, the LUMINA, the Toronto and the SLICC studies included cerebrovascular and peripheral vascular atherosclerotic vascular events while the Baltimore and Pittsburgh studies included only coronary artery disease events. While there were no cerebrovascular accidents reported in the current study there were two instances of transient ischemic attack and four with peripheral vascular disease. This difference in outcomes might have an effect on the nature of the associated factors.

We have previously demonstrated in this cohort that risk factors for atherosclerotic disease accumulate early in the course of the disease ${ }^{17}$. However as suggested earlier, these risk factors have not yet exerted their full impact. In early disease it is likely that male gender and older age states associated with increased risk factors for atherosclerotic vascular events are dominant. This should not deter from the necessity for aggressive intervention in the treatment of classic CAD risk factors.

Atherosclerotic vascular events documented in this cohort included cardiac, cerebrovasular and peripheral vascular events. Cardiac and cerebral events are common disease features of SLE. These clinical presentations often pose a diagnostic challenge as they are often confounded by co-morbid conditions. As well atherosclerotic events may mimic SLE presentations for example chest pain and stroke syndromes can be on the basis of active SLE or atherosclerosis. Furthermore, this is made more difficult by the fact that the patients who presented with atherosclerotic vascular events early in the course of their SLE disease had similar risk factors to patients who presented with a lupus vascular event except with regards to older age at diagnosis, Caucasian race and use of antimalarials. Unlike other studies where antimalarials have been suggested to be protective for atherosclerotic or thrombotic disease, in the current study patients with atherosclerotic events more often took antimalarials than those who did not have atherosclerotic events ${ }^{18,19,20}$. The reason for this is unclear. While it is possible that patients with atherosclerotic events had had more severe disease prior to enrolling in the SLICC-RAS, at two years, patients without atherosclerotic events actually had higher disease activity measured by the AMS. Thus the reason for the lower use of antimalarials in these patients is unknown. Clinicians must adopt attribution rules, as used in this study, to differentiate those events due to the disease itself from those due to atherosclerosis. In addition to the clinical attribution rules, most patients in this study had confirmatory laboratory and imaging studies. 
A limitation of this study is the relatively short duration of follow-up of an inception cohort and therefore the number of events is small. Nevertheless, 31 events in 22 patients were detected within 2.5 years of diagnosis, highlighting the fact that in some individuals (older males) these events may occur very early in the disease. Another possible limitation is the interval between assessments being one year, which may not reveal the full impact of disease activity on the development of atherosclerotic events. The strengths of this study are the size of the inception cohort, its multiethnic composition, the uniform collection of data on a common data retrieval protocol, and the use of standardized clinical attribution rules for atherosclerotic vascular events. Nevertheless in patients with known atherosclerotic disease active lupus may make the attribution of an event more difficult, and in cases of active lupus subclinical atherosclerosis may be missed. However, in the majority of cases the attribution rules helped clarify the situation. Patients in this cohort will continue to be followed for 5 years.

\section{Acknowledgments}

We are grateful for the generous donation of our patients' time and the dedication of all the fellows, research coordinators and research assistants in the SLICC network to the completion of this work.

Support: Dr. Urowitz's work was supported by the Canadian Institutes of Health Research (grant MOP-49529), the Lupus Foundation of Ontario, the Ontario Lupus Association, Lupus UK, the Lupus Foundation of America, the Lupus Alliance of Western New York, the Conn Smythe Foundation, The Lupus Flare Foundation, and the Tolfo family of Toronto, Ontario, Canada. Dr. Bae's work was supported by the Brain Korea 21 Program. Dr. Gordon's work was supported by Lupus UK, the Arthritis Research Campaign, and the Wellcome Trust Clinical Research Facility, Birmingham, UK. Dr Alarcón's work was supported by the University of Alabama at Birmingham (NIH/ NIAMS grant P60-AR-48095). Dr. Clarke's work was supported by the Singer Family Fund for Lupus Research; she is also a Fonds de la Recherche en Sante du Quebec National Scholar. Dr. Bernatsky's work was supported by the Fonds de la Recherche en Sante du Quebec Jeune Chercheure and the McGill University Health Centre Research Institute; she is also a recipient of a Canadian Institutes of Health Research Junior Investigator Award and a Canadian Arthritis Network Scholar Award. Dr. Fortin is a Distinguished Senior Investigator of The Arthritis Society with additional support from the Arthritis Centre of Excellence, University of Toronto. Dr. Hanly's work was supported by a grant from the Canadian Institutes of Health Research. Dr. Gladman's work was supported by the Canadian Institutes of Health Research. Dr. Petri's work was supported by the Hopkins Lupus Cohort (grant AR-43727) and the Johns Hopkins University General Clinical Research Center (grant M01-RR-00052). Drs. Nived and Sturfelt's work was supported by the Swedish Medical Research Council (grant 13489). Dr. RamseyGoldman's work was supported by the NIH (grants UL 1RR 025741, K24-AR-02318, and P60-AR-48098).

\section{References}

1. Urowitz MB, Bookman AAM, Koehler BE, Gordon DA, Smythe HA, Ogryzlo MA. The Bimodal Mortality in Systemic Lupus Erythematosus. Am J Med. 1976; 60:221-225. [PubMed: 1251849]

2. Petri M, Perez-Gutthan S, Spence D, Hochberg MC. Risk factors for coronary artery disease in patients with systemic lupus erythematosus. Amer J Med. 1992; 93:513-519. [PubMed: 1442853]

3. Manzi S, Meilahn EN, Rairie JE, et al. Age-specific incidence rates of myocardial infarction and angina in women with Systemic Lupus Erythematosus: comparison with the Framingham study. Amer J Epidemiol. 1997; 145:408-15. [PubMed: 9048514]

4. Gladman DD, Urowitz MB. Morbidity in systemic lupus erythematosus. J Rheumatol. 1987; 14(Suppl 13):223-6.

5. Manzi S, Selzer F, Sutton-Tyrrel K, Fitzgerald S, Rairie JE, Tracy RP, Kuller LH. Prevalence and risk factors of carotid plaque in women with systemic lupus erythematosus. Arthritis Rheum. 1999; 42:51-60. [PubMed: 9920014]

6. Isenberg DA, Gladman DD. The Systemic Lupus International Collaborating Clinics Group Origins and outcomes. Lupus. 2001; 10:375-77. [PubMed: 11403271]

7. Urowitz MB, Gladman D, Ibañez D, Fortin P, Sanchez-Guerrero J, Bae S, et al. Clinical manifestations and coronary artery disease risk factors at diagnosis of Systemic Lupus Erythematosus: Data from an international inception cohort. Lupus. 2007; 16:731-735. [PubMed: 17728367] 
8. Hochberg MC. Updating the American College of Rheumatology revised criteria for the classification of systemic lupus erythematosus. Arthritis Rheum. 1997; 40:1725. [PubMed: 9324032]

9. Gladman DD, Ibañez D, Urowitz MB. SLE Disease Activity Index 2000. J Rheumatol. 2002; 29:288-291. [PubMed: 11838846]

10. Ibañez D, Urowitz MB, Gladman DD. Summarizing disease features over time: I. Adjusted mean SLEDAI derivation and application to an index of disease activity in lupus. J Rheumatol. 2003; 30:1977-1982. [PubMed: 12966601]

11. Gladman DD, Ginzler E, Goldsmith C, Fortin P, Liang M, Urowitz M, Bacon P, et al. Development and Initial Validation of the SLICC/ACR Damage Index for SLE. Arthritis Rheum. 1996; 39:363-9. [PubMed: 8607884]

12. Gladman DD, Urowitz MB, Goldsmith C, Fortin P, Ginzler E, Gordon C, et al. The Reliability of the SLICC/ACR Damage Index in Patients with SLE. Arthritis Rheum. 1997; 40:809-13. [PubMed: 9153540]

13. Tustall-Pedoe H, Kuulasmaa K, Abouyel P, Arveiler D, Rajakangas AM, Pajak A. Myocardial infarction and coronary deaths in the World Health Organization MONICA project. Registration procedures, event rates, and case-fatality rates in 38 populations from 21 countries in four continents. Circulation. 1994; 90:583-612. [PubMed: 8026046]

14. Nikpour M, Urowitz MB, Gladman DD. Premature atherosclerosis in systemic lupus erythematosus. Rheum Dis Clin North Am. 2005; 31:329-354. [PubMed: 15922149]

15. Urowitz MB, Ibañez D, Gladman DD. Atherosclerotic vascular events (AVE) in a single large lupus cohort: prevalence and risk factors. J Rheumatol. 2007; 34:70-75. [PubMed: 17117488]

16. Toloza SM, Uribe AG, McGwin G Jr, Alarcon GS, Fessler BJ, Bastian HM, et al. Systemic lupus erythematosus in a multiethnic US cohort (LUMINA). XXIII. Baseline predictors of vascular events. Arthritis Rheum. 2004; 50:3947-3957. [PubMed: 15593203]

17. Urowitz MB, Gladman D, Ibañez D, Fortin P, Sanchez-Guerrero J, Bae S, et al. Accumulation of coronary artery disease risk factors over three years: data from an international inception cohort. Arthritis Rheum. 2008; 59:176-180. [PubMed: 18240193]

18. Urowitz MB, Gladman DD, Tom BDM, Ibañez D, Farewell VT. Changing Patterns in Mortality and Disease Outcome for Patients with Systemic Lupus Erythematosus. J Rheumatol. 2008; 35:2152-2158. [PubMed: 18793004]

19. Alarcon GS, McGwin G, Bertoli AM, et al. Effect of hydroxychloroquine on the survival of patients with systemic lupus erythematosus: data from LUMINA, a multiethnic US cohort. Ann Rheum Dis. 2007; 66:1168-72. [PubMed: 17389655]

20. Ruiz-Irastorza G, Egurginde MB, Pijoan JI, et al. Effect of antimalarials on thrombosis and survival in patients with systemic lupus erythematosus. Lupus. 2006; 15:577-83. [PubMed: 17080912] 
Table 1

Characteristics of the SLICC-RAS cohort at enrolment $(n=1249)$

\begin{tabular}{|l|c|}
\hline Gender - Female - n (\%) & $1117(89.4 \%)$ \\
\hline Race/Ethnicity - (\%) Caucasian / Black / Hispanic / Asian/Other & $49 / 15 / 16 / 16 / 4$ \\
\hline Age at diagnosis - years & $34.3 \pm 13.3$ \\
\hline Disease duration - months & $5.5 \pm 4.1$ \\
\hline SLEDAI-2K - mean \pm sd & $5.49 \pm 5.59$ \\
\hline Corticosteroids - n (\%) & $849(69.7 \%)$ \\
\hline Immunosuppressives - n (\%) & $485(39.8 \%)$ \\
\hline Antimalarials - n (\%) & $771(63.3 \%)$ \\
\hline
\end{tabular}


Table 2

Attribution of vascular events

\begin{tabular}{|l|c|c|c|}
\hline & Attributed to Atherosclerosis & Attributed to Active SLE & Attributed to Other Causes* $^{*}$ \\
\hline Number of events & 31 & 50 & 16 \\
\hline Stroke & & 20 & 3 \\
\hline Congestive Heart Failure & 5 & 12 & 7 \\
\hline Transient Ischemic Attack & 2 & 10 & 1 \\
\hline Peripheral Vascular Disease & 4 & 3 & 1 \\
\hline Angina & 12 & 2 & 3 \\
\hline Myocardial Infarction & 8 & 2 & \\
\hline Pacemaker Insertion & & 1 & \\
\hline
\end{tabular}

* List of Causes

Coagulopathy 3

Pregnancy complications 2

Fluid overload 2

Post-operative complications 1

Vertebral artery dissection 1

Pulmonary disease 1

Gastrointestinal bleed 1

Renal failure on dialysis 1

Coronary artery spasm 1

Hypertension 1

Unknown 2 
Table 3

A comparison between patients with atherosclerotic vascular events due to atherosclerosis with all other patients

\begin{tabular}{|l|c|c|c|}
\hline At Enrolment & Patients with AVE & All other Patients & P value \\
\hline No. of patients & 22 & 615 & \\
\hline Caucasian & $17(77.3 \%)$ & $328(53.3 \%)$ & 0.03 \\
\hline Male & $9(40.9 \%)$ & $72(11.7 \%)$ & $<0.0001$ \\
\hline Age at Diagnosis & $54.6 \pm 13.1$ & $34.6 \pm 13.7$ & $<0.0001$ \\
\hline Hypertension & $14(73.6 \%)$ & $197(32.3 \%)$ & 0.002 \\
\hline Obese (BMI >27 F >25 M) & $12(57.1 \%)$ & $163(28.1 \%)$ & 0.004 \\
\hline Smoker (Current or Ex) & $14(63.6 \%)$ & $225(36.6 \%)$ & 0.01 \\
\hline Family History CAD (adjusted for age) $* *$ & $9(42.9 \%)$ & $117(20.0 \%)$ & 0.02 \\
\hline Hypercholesterolemia & $12(54.6 \%)$ & $228(37.4 \%)$ & 0.10 \\
\hline Diabetes mellitus & $2(10.5 \%)$ & $14(2.4 \%)$ & 0.08 \\
\hline Corticosteroids & $17(77.3 \%)$ & $417(68.5 \%)$ & 0.38 \\
\hline Antimalarials & $16(72.7 \%)$ & $386(63.5 \%)$ & 0.38 \\
\hline Immunosuppressive drug use & $6(27.3 \%)$ & $227(37.3 \%)$ & 0.34 \\
\hline SLEDAI-2K at presentation & $3.32 \pm 3.09$ & $5.53 \pm 5.63$ & 0.11 \\
\hline AMS at Year 2 & $3.02 \pm 3.11$ & $4.15 \pm 3.78$ & 0.12 \\
\hline
\end{tabular}

Followed for at least 2 years, AMS=adjusted mean SLEDAI-2K

**

first degree relative Female $<65$ years old or Male $<55$ years old 
Table 4

A comparison between patients with vascular events due to atherosclerosis to patients with vascular events due to SLE.

\begin{tabular}{|l|c|c|c|}
\hline & Patients with vascular events due to AS & Patients with vascular events due to SLE & P value \\
\hline No. of patients ${ }^{*}$ & 22 & 42 & \\
\hline Caucasian & $17(77.3 \%)$ & $21(50.0 \%)$ & 0.03 \\
\hline Male & $9(40.9 \%)$ & $8(19.1 \%)$ & 0.06 \\
\hline Age at Diagnosis & $54.6 \pm 13.1$ & $41.2 \pm 13.8$ & 0.0004 \\
\hline Hypertension & $14(63.6 \%)$ & $28(66.7 \%)$ & 0.81 \\
\hline Obese (BMI >27 F >25 M) & $12(57.1 \%)$ & $15(36.6 \%)$ & 0.12 \\
\hline Diabetes mellitus & $2(10.5 \%)$ & $1(2.4 \%)$ & 0.23 \\
\hline Smoker (Current or Ex) & $14(63.6 \%)$ & $16(38.1 \%)$ & 0.052 \\
\hline Family Hx CAD (adjusted for age) $* *$ & $9(42.9 \%)$ & $13(31.7 \%)$ & 0.39 \\
\hline Hypercholesterolemia & $12(54.6 \%)$ & $19(45.2 \%)$ & 0.48 \\
\hline Antiphospholipid Ab at enrolment ${ }^{* * *}$ & $8 / 20(40.0 \%)$ & $15 / 35(42.9 \%)$ & 0.84 \\
ACL & $3 / 17(17.7 \%)$ & $14 / 32(15.6 \%)$ & 0.00 \\
\hline LAC & $6 / 20(30.0 \%)$ & $30(71.4 \%)$ & 0.46 \\
\hline Antimalarials & $17(77.3 \%)$ & $15(36.6 \%)$ & 0.62 \\
\hline Immunosuppressive drug use & $16(72.7 \%)$ & $21(50.0 \%)$ & 0.006 \\
\hline SLEDAI-2K & $6(27.3 \%)$ & $5.93 \pm 7.47$ & 0.08 \\
\hline AMS at Year 2 & $3.32 \pm 3.09$ & $4.91 \pm 4.82$ & 0.43 \\
\hline
\end{tabular}

There were 31 atherosclerotic vascular events in 22 patients, and 50 vascular events due to SLE in 42 patients.

** first degree relative Female $<65$ years old or Male $<55$ years old.

*** anticardiolipin antibody or lupus anticoagulant AMS=adjusted mean SLEDAI-2K 


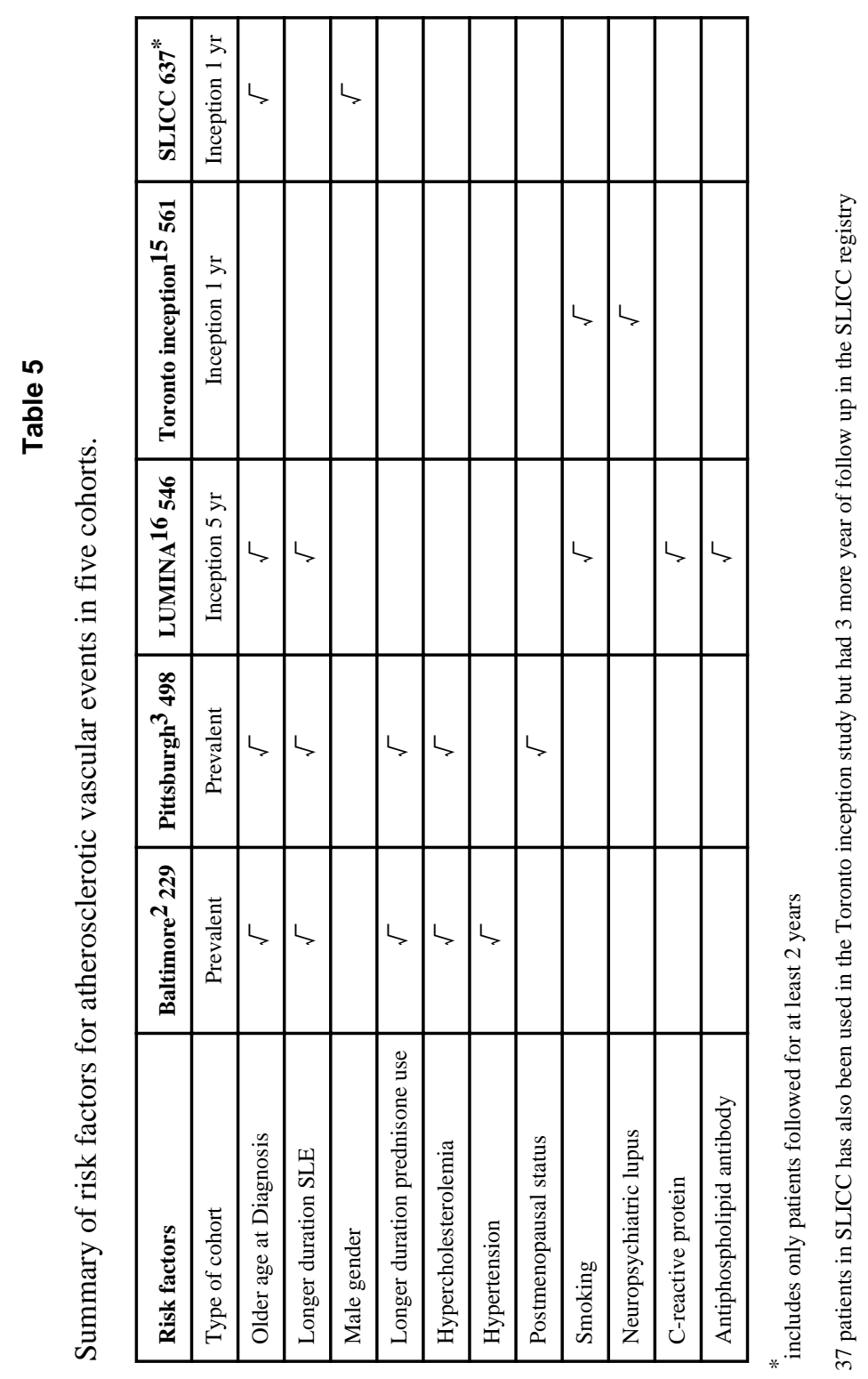

Arthritis Care Res (Hoboken). Author manuscript; available in PMC 2011 June 1. 